professionals who were injured were not using gloves when administering medication, and $15.3 \%$ and $14.7 \%$, respectively, did not do so in the procedures of blood collection and laboratory collection. Glove use during accident in surgical and dental procedures occurred in $96.7 \%$ and $95.3 \%$, respectively. The outpatient discharge of the injured professionals occurred in $39.6 \%(49,557)$ after confirming a negative source for hepatitis B, C and HIV (human immunodeficiency virus), in $22.1 \%(27,674)$ after six months of follow-up without serological conversion and in $11.6 \%$ due to abandonment of follow-up. In this period there were no cases of conversion to HIV.

Conclusion Reporting accidents with biological material is a great monitoring tool. Inadequate disposal of needles is an important cause of accidents, and their frequency remains unchanged over the years. The accidents in procedures of realisation of blood test and recapping needles had a decrease. Much more can be done to prevent accidents and improve the follow-up of accidents.

\section{P3.234 CHLAMYDIA TRACHOMATIS: GEOGRAPHICAL VARIATION IN TEST PRACTICES OF GENERAL PRACTITIONERS, 2011-2015}

\begin{abstract}
${ }^{1,2}$ JAP Wijers, ${ }^{1,2}$ GAFS van Liere, ${ }^{1,2}$ CJPA Hoebe, ${ }^{1,2}$ NHTM Dukers-Muijrers. ${ }^{1}$ Department of Sexual Health, Infectious Diseases and Environment, Public Health Service South Limburg, Geleen, South Limburg, The Netherlands; '2Department of Medical Microbiology, Care and Public Health Research Institute (CAPHRI), Maastricht University Medical Centre (MUMC+), Maastricht, South Limburg, The Netherlands
\end{abstract}

10.1136/sextrans-2017-053264.469

Introduction Retesting Chlamydia trachomatis (CT) treated people after 3-12 months is recommended as it can yield substantial numbers of reinfections. A test-of-cure (TOC) shortly after treatment (within 3 months) is not advisable due to the likelihood of false positive findings leading to overtreatment. Spatial analyses are useful to detect geographical areas of low guideline adherence to inform local testing policies and targeted interventions. The aim was to assess geographical variation in test practices of general practitioners (GPs) in The Netherlands.

Methods Retrospective laboratory data containing CT tests of 48 GPs in 4 municipalities were obtained (2011-2015) from the public laboratory in the south western part of the Netherlands (183 thousand residents). First recorded urogenital positive CT tests of men $(n=249 ; 39.2 \%)$ and women $(n=386 ; 60.8 \%) \geq 16$ years between January 2011 and July 2015 were included in the analyses and TOC and retests were outcomes. Logistic regression was used for analyses.

Results Overall, 8,275 CT tests were performed (positivity $8.4 \% ; n=691)$; only $0.4 \%(n=43)$ from extra genital sites. On a GP level, the number of CT tests varied geographically from 1 to $2421(\mathrm{p}<0.001)$. A TOC was performed in $19.1 \%$ of the CT cases $(n=123 ; 13.8 \%$ positive). TOC was more often performed in south Maastricht in comparison with the centre of Maastricht $(\mathrm{p}=0.02$,OR 3.0,95\% CI 1.23-7.33). A retest was performed in $23 \%$ of the CT cases $(n=146 ; 10.3 \%$ positive). The rate of retests non-significantly varied geographically between $6.3 \%$ and $30.2 \% \mathrm{p}=0.33$. Patients with a TOC were more likely to have a retest in comparison with cases without a TOC $(\mathrm{p}=0.02)$.

Conclusion Testing at extra genital sites and the overall proportion of retests was low at GP practices. Almost one out of five CT cases returned within three months, and many (re-) infections were probably missed. Moreover, it seems that there are geographical variations in test practices of GPs. Thus, targeted interventions at the local level are needed to increase CT testing and retesting practices of GPs.

\section{P3.235 PARENTAL ACCEPTANCE OF HPV VACCINE IS HIGH AND BASED ON POOR KNOWLEDGE}

${ }^{1}$ WM Lobão, ${ }^{1}$ CAST Santos, ${ }^{1}$ GAC Santos, ${ }^{1}$ MCC Almeida, 1,2ED Moreira Jr. ' Gonçalo Moniz Institute, Oswaldo Cruz Foundation, Brazil; ${ }^{2}$ Clinical Research Centre, Associação Obras Sociais Irmã Dulce, Brazil

\section{$10.1136 /$ sextrans-2017-053264.470}

Introduction Vaccination coverage levels of the National Immunisation Program (PNI) in Brazil ranges from $80 \%$ to $95 \%$, suggesting that parental acceptance of these vaccines is high. In 2014, HPV vaccine was introduced in the PNI. We conducted a survey to estimate parental acceptance of HPV vaccine and its determinants.

Methods This was a random digit calling telephone survey performed in seven large cities from all five regions in Brazil. Eligible parents had to have one or more child less than 18 years old. We selected at least 100 subjects in each city. A standardised questionnaire was used to collect sociodemographic informationa and data on knowledge, attitudes and practices related to HPV vaccine, cervical cancer and HPV. Trained personnel conducted the interviews that lasted on average $20 \mathrm{~min}$.

Results Overall 826 parents were included in the survey $(73 \%$ response rate). Parental acceptance for vaccinating against HPV was similarly high for female or male children, $92.8 \%$ and $91.7 \%$, respectively. It did not change significantly among the cities studied (range 86.3\%-95.5\%). Parents's main reasons to give the HPV vaccine to their children were: "Vaccines are good/important"(85.6\%), "Prevents cervical cancer"(6.6\%), and "Vaccine is in the PNI"(3.3\%). Only 0.7\% reported "Prevents genital warts". Among parents with girls eligible for HPV vaccination (10-14 years old) under the PNI $(n=291), 71.4 \%$ had their daughters vaccinated.

Conclusion Parental acceptance of HPV vaccine is high (92\% $\%$ ), but a lower percentage results in actual vaccination. Moreover, the main reason to vaccinate is based on a vague assumption ("vaccines are good/important"). Only few parents reported that HPV vaccination prevents cervical cancer. Parents whose vaccine acceptance is ill founded and based on poor knowledge are more vulnerable to change their mind when challenged by stress related mass reactions to HPV vaccine reported by the media or when facing false arguments against vaccination by anti-vax reports.

\section{P3.236 STUDY OF GENITAL CANCER AETIOLOGY: ASSOCIATION OF HUMAN PAPILOMAVIRUS (HPV) AND MERKEL CELL POLYOMAVYRUS (MCPYV)}

${ }^{1}$ Rocha WM, ${ }^{2} \mathrm{CF}$ Baez, ${ }^{1} \mathrm{LA}$ Afonso, ${ }^{1} \mathrm{FN}$ Carestiato, ${ }^{1} \mathrm{RB}$ Varella, ${ }^{1} \mathrm{SMB}$ Cavalcanti. ${ }^{1}$ Universidade Federal Fluminense - Instituto Biomédico - Laboratório de Diagnóstico Virológico, Brazil; ${ }^{2}$ Universidade Federal do Rio de Janeiro - Departamento de Medicina Preventiva, Brazil

10.1136/sextrans-2017-053264.471 
Introduction The genital infection by the HPV is among the most frequent sexually transmitted diseases (STD) worldwide, and it may result in lesions that can lead to the carcinogenesis of the genital tract. However, other factors may be associated with the onset or progression of the tissue malignancy process, such as the MCPyV, which may present oncogenic profile in the epithelial tissue. This study aims to investigate the presence of MCPyV and HPV in malignant lesions of the male and female genital tract, in order to contribute to the elucidation of the role of these viruses in the cellular malignancy process and to the epidemiological knowledge regarding the prevalence of both viruses in neoplastic lesions.

Methods This is a cross-sectional study evaluating the prevalence of HPV and MCPyV infection in samples of cervical carcinoma and penile cancer. To date, we have obtained 112 samples of penile carcinoma and 31 samples of cervical carcinoma. So, we aim to detect the presence of HPV DNA by the polymerase chain reaction (PCR) technique using the generic primers MY09/MY11; to genotype HPVs by specific PCR to the E6 gene; to detect and quantify DNA of the MCPyV by the Nested PCR technique and real-time PCR; to investigate the presence of truncation mutations in the major $\mathrm{T}$ antigen of MCPyV.

Results Results are partial. To date, all the male samples were analysed. We verified the presence of HPV in $54(48.2 \%)$ of these samples, in which the most prevalent type was the HPV16 (66\%). The cervical carcinoma samples are still under analysis.

Conclusion The collection of cervical neoplasia samples is still being performed. In 2015, our research group found a case of multiple infection by HPV, MCPyV and Epstein-Barr virus in a case of squamous cell carcinoma of the penis in Rio de Janeiro. This was the first report of the presence of MCPyV in this type of penile lesion. Thus, we look forward to find results that contribute to the presence of $\mathrm{MCPyV}$ in genital malignant lesions and to elucidate its role in the oncogenic pathway of malignant lesions.

\section{P3.237 DIRECT DETECTION OF MOSAIC PENA IN CLINICAL SAMPLES CONTAINING NEISSERIA GONORRHOEAE}

${ }^{1}$ PFG Wolffs, 'BMJW van der Veer, 1,2CJPA Hoebe, ${ }^{1,2}$ Dukers-Muijrers NHTM, '1van Alphen LB, ${ }^{1} \mathrm{IHM}$ van Loo. ${ }^{1}$ Maastricht University Medical Centre, Care and Public Health Research Institute (CAPHRI), Maastricht, the Netherlands; ${ }^{2}$ Public Health Service South Limburg, Department of Sexual Health, Infectious Diseases and Environmental Health, Geleen, the Netherlands

\subsection{6/sextrans-2017-053264.472}

Introduction Current surveillance of antibiotic resistance in Neisseria gonorrhoeae (NG) relies heavily on the culture of NG. However, culture of NG is challenging due to demanding nutritional and growth requirements of this micro-organism. As a result, surveillance data are limited to only cultured strains while of $>50 \%$ of Dutch NG positive patients no NG is cultured (data from Dutch Gonococcal Surveillance Program). In this study we compared results from direct detection of mosaic penA with detection of cultured strains to investigate feasibility of direct molecular resistance surveillance.

Methods A convenience sample of 106 NG positive samples of which positive NG culture results were available (46 urine, 9 genital swabs, 35 anorectal swabs and 16 oropharyngeal swabs) were collected between 2013-2015. Presence of mosaic penA was determined by real-time PCR. All positive findings were confirmed with sequencing. MICs on cultured NG were determined using E-tests.

Results LOD determinations of the in-house mosaic penA PCR in comparison to routine NAAT (using COBAS 4800, Roche Diagnostics) showed that the mosaic penA assay was slightly less sensitive than the commercial NAAT. In samples with very low NG loads, mosaic penA detection might be false-negative. Of $106 \mathrm{NG}$ positive samples, 11 samples showed the presence of mosaic penA (6 urine, 4 oropharyngeal and 1 anorectal swab). Of these 11 samples, NG isolates were re-cultured from 8 samples and all isolates contained the mosaic penA gene. MIC values for ceftriaxone varied between 0.016 and $0.094 \mathrm{mg} / \mathrm{L}$ and thus no reduced susceptibility was observed. Although cross-detection with mosaic penA from $N$. meningitidis is possible, no evidence of this was shown in this study.

Conclusion In conclusion, this study indicates that detection of mosaic penA directly from clinical samples is feasible and that results match detection of penA from clinical isolates obtained from these samples. Direct detection of antibiotic resistance genes would show an insight in resistance surveillance of strains that are not or cannot be cultured.

\section{P3.238 SYPHILIS INFECTION STATUS AND THE ASSOCIATED FACTORS AMONG PARTNERS OF SYPHILIS-INFECTED PREGNANT WOMEN IN SHENZHEN, CHINA}

$X$ Wu, F Yang, L Lan, Y Guan, C Zhang, T Feng. Shenzhen Centre for Chronic Disease Control, Shenzhen, China

\subsection{6/sextrans-2017-053264.473}

Introduction The goal of this study is to investigate syphilis infection status among partners of syphilis-infected pregnant women, and to analyse the associated factors from the perspective of both pregnant women and their partners.

Methods This study was based on Shenzhen Program for Prevention of Mother-to-Child Transmission of Syphilis. Followup survey and contact tracing were conducted among syphilisinfected pregnant women diagnosed from 2008 to 2015. Both toludine red unheated serum test (TRUST) and treponema pallidum particle agglutination (TPPA) were applied to partners attending antenatal care clinics. Structural questionnaire was used to collect information. Binary logistic regression model was applied to analyse the factors associated with partners' syphilis infection.

Results During the studied years, 6318 pregnant women were identified with syphilis infection and 5351 partners of these women visited the antenatal care clinics and took the serological testing. From the laboratory record and clinical examination results, 1148 partners were diagnosed with syphilis infection. The infection rate decreased from $26.23 \%$ in 2008 to $15.07 \%$ in 2015 . The partners were more likely to have syphilis infection when pregnant women with TRUST titers $\geq 1$ 8 or at the early syphilis stage. The partners had more risk of syphilis infection when they had multiple sex partners.

Conclusion Syphilis infection rate among partners of syphilisinfected pregnant women is high. Pregnant women's levels of TRUST titer and disease stage as well as partners' sexual behaviours were important factors associated with partners' syphilis infection. Partner notification and contact tracing is an important way to identify syphilis patients. 